\section{Redesigning services for the management of vitreomacular traction and macular hole}

W Amoaku' ${ }^{1}$ P Cackett ${ }^{2}$, A Tyagi ${ }^{3}$, U Mahmood ${ }^{4}$, J Nosek ${ }^{5}$, G Mennie ${ }^{6}$ and N Rumney ${ }^{7}$
${ }^{1}$ Faculty of Medicine and Health Sciences, Queen's Medical Centre, University of Nottingham, Nottingham, UK

Vitreomacular traction (VMT) occurs when a persistent vitreomacular adhesion (VMA) exerts tractional stress on the macula, leading to deformation; VMT itself may be associated with macular hole $(\mathrm{MH})(\mathrm{VMT}+\mathrm{MH}$; Figure 1$) .{ }^{1}$ $\mathrm{VMT}$ and VMT $+\mathrm{MH}$ are serious conditions, being associated with visual disturbance, for example, metamorphopsia, and diminished visual acuity (VA). ${ }^{2-6}$ Consequently, they are also known to exert a significant negative impact on the patients' quality of life and their ability to perform daily tasks. ${ }^{7,8}$ The risk of VMT and $\mathrm{VMT}+\mathrm{MH}$ increases with age - the median age of patients is around 65-69 years. $^{9-11}$

The prevalence of isolated VMT has been estimated at around 22.5 per 100000 of the general population. ${ }^{1}$ This compares with an estimated prevalence of 140-149/100000 and an annual incidence of 7.8-8.8/100000 for $\mathrm{VMT}+\mathrm{MH} .{ }^{1,2,9}$ In 2012-2013, it was anticipated that there would be more than 17000 vitrectomies performed in England. Of these, an estimated $42 \%$ were for $\mathrm{VMT}+\mathrm{MH}$ and $9 \%$ were for VMT. ${ }^{12}$

\section{Treatment of VMT}

A period of observation ('watchful waiting') of at least 3 months is generally employed prior to intervention in patients with VMT, with the aim of allowing spontaneous resolution. ${ }^{13}$ However, only around $10 \%$ of VMT cases resolve spontaneously, and the timeframe for this is years rather than months. ${ }^{14}$ Indeed, VMT tends to progress over time (with the development of $\mathrm{MH}$ or persistent cystoid changes), accompanied by deteriorating VA. ${ }^{14}$
${ }^{2}$ Princess Alexandra Eye Pavilion, Edinburgh, UK

${ }^{3}$ Birmingham and Midlands Eye Centre and Sandwell General Hospital, Birmingham, UK

${ }^{4}$ University Hospital of North Staffordshire, Stoke-onTrent, UK

${ }^{5}$ Wolverhampton Eye Infirmary, Wolverhampton, UK

${ }^{6}$ Gloucestershire Clinical Commissioning Group, Brockworth, UK

${ }^{7}$ BBR Optometry, Hereford, UK

Correspondence: W Amoaku, Queen's Medical Centre, Room B Floor Eye and ENT Centre, University Hospital, Nottingham NG7 2UH, UK Tel: + 44 (0)115 951 5151; Fax: + 44 (0)115962 7765 E-mail: Winfried.Amoaku@ nottingham.ac.uk

Please note that prescribing information is available at the end of this article as an Appendix.

VMT Service Re-Design Advisory Board Meeting, Birmingham, UK, 20 September 2013. 

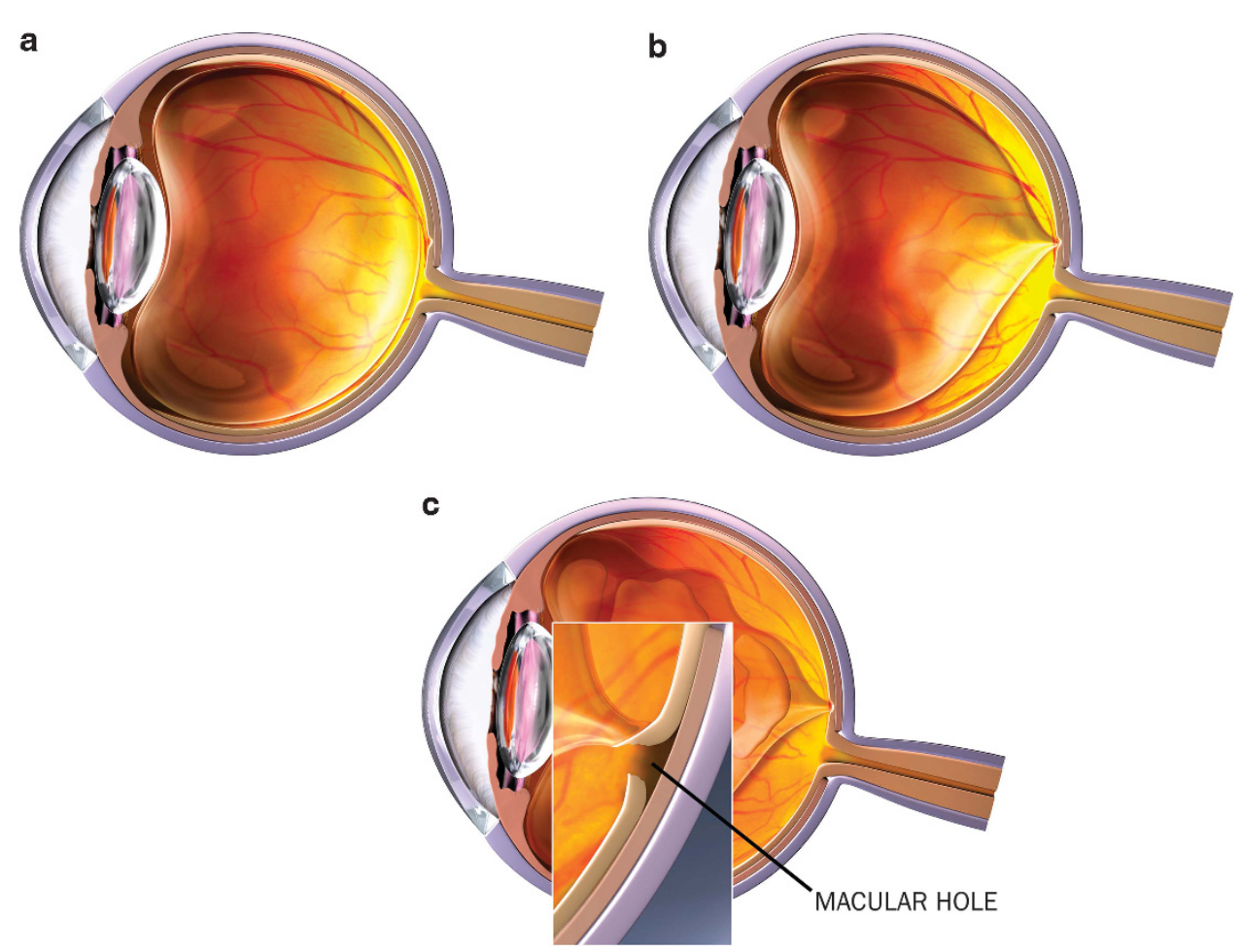

Figure 1 Visual representation of (a) normal vision, (b) vitreomacular traction, and (c) macular hole.

Furthermore, it has been demonstrated that improvements in VA following vitrectomy for VMT are better in patients with a shorter duration of symptoms prior to surgery. ${ }^{15}$

Pars plana vitrectomy (PPV) is the routine treatment for symptomatic VMT, performed with the aim of releasing the residual VMA in order to restore normal central retinal architecture. ${ }^{1,13}$ In a study of 20 eyes with VMT, 16 achieved an increase in VA of $\geq 2$ Snellen lines; 1 eye developed an $\mathrm{MH} .^{16}$

\section{Treatment of VMT with MH}

A small minority of $\mathrm{VMT}+\mathrm{MH}$ cases may resolve spontaneously (approximately 10\%). ${ }^{17,18}$ This means that almost all cases of VMT $+\mathrm{MH}$ progress over time, with an attendant deterioration in VA. ${ }^{17-19}$

PPV is the current standard of care for VMT $+\mathrm{MH}^{1,13}$ One study utilising modern techniques reported an $\mathrm{MH}$ closure rate of $84 \%,{ }^{20}$ a large review of the National Ophthalmology Database (NOD) reported VA improvement of $\geq 2$ Snellen lines in $49 \%$ of eyes at 12 weeks after surgery for VMT $+\mathrm{MH}^{21}$

It has been demonstrated that delaying vitrectomy is associated with worse outcomes compared with prompt intervention. ${ }^{10,22}$ One study showed that, in patients with $\mathrm{VMT}+\mathrm{MH}$ of $\leq 6$ months' duration, successful closure was achieved in $95 \%$ of patients compared with $47 \%$ in patients with VMT $+\mathrm{MH}$ duration $>1$ year. ${ }^{11}$ In addition, postoperative VA of 6/12 Snellen lines or better was achieved in $52 \%$ of patients with VMT $+\mathrm{MH}$ duration of 6 months or less, compared with $16 \%$ of patients with $\mathrm{MH}$ of more than 1 year. The probability of successful MH closure decreased by $6 \%$ with each month of delay prior to surgery. ${ }^{11}$ In addition, specific to VMT without $\mathrm{MH}$, it has been suggested that excessive traction upon the fovea and its deformation can lead to structural changes that may not recover completely, either anatomically or functionally, after vitreous release. ${ }^{23}$ In one study, 69 cases of VMT that had recently converted from VMA were observed over a 2-year period; eight developed epiretinal membrane (ERM), six of which underwent vitrectomy with symptoms of reduced VA and metamorphopsia, five underwent cataract surgery, two developed $\mathrm{MH}$, and one developed lamellar $\mathrm{MH}$, whereas one patient had surgery to remove a dropped nucleus. ${ }^{23}$ The evolving evidence therefore suggests that observing VMT may result in progression of vitreomacular interface diseases, such as ERM, which can negatively impact success rates of subsequent intervention, highlighting the importance of considering early intervention in this group of symptomatic patients.

Even with modern approaches, PPV may be associated with retinal detachment $(7 \%)$, chronic intraocular pressure elevation $(6 \%)$, and cystoid macular oedema $(5 \%) .{ }^{24}$ In the NOD study, $4.2 \%$ of eyes with $\mathrm{MH}$ 
underwent repeat PPV. ${ }^{21} \mathrm{~A}$ recent study has reported a need for subsequent cataract surgery in approximately $50-65 \%$ of patients within 1 year of PPV for $\mathrm{MH}^{21,25}$

\section{A new paradigm in VMT treatment}

Ocriplasmin was approved for use in the European Union in patients with VMT, including in association with $\mathrm{MH}$ ( $\leq 400 \mu \mathrm{m}$ diameter) in March 2013. ${ }^{26}$ This followed the completion of two separate Phase III randomised, double-blind, placebo-controlled clinical trials that were performed to evaluate the efficacy and safety of a single intravitreal injection of ocriplasmin $125 \mu \mathrm{g}$ in patients with VMT and/or $\mathrm{MH}(\leq 400 \mu \mathrm{m}$ in diameter). ${ }^{27}$ After 28 days the proportion of patients with VMA resolution was significantly higher with ocriplasmin compared with placebo (26.5 vs 10.1\%; $P \leq 0.001$ ), and the difference between groups was significant from day 7 after treatment. Complete posterior vitreous detachment (PVD) was achieved in $13.4 \%$ of eyes receiving ocriplasmin and $3.7 \%$ of patients receiving placebo $(P \leq 0.001) .{ }^{27}$ Closure of $\mathrm{MH}$ was observed in $40.6 \%$ of eyes in the ocriplasmin group and in $10.6 \%$ of eyes in the placebo group $(P \leq 0.001)$. Smaller MHs closed more frequently than larger ones, with a $58.3 \%$ closure rate in $\mathrm{MH} \leq 250 \mu \mathrm{m}$ and $24.6 \%$ closure in holes $250-400 \mu \mathrm{m}$ in diameter. In addition, successful resolution of VMA with ocriplasmin was achieved in fewer eyes demonstrating an ERM than in the absence of ERM (8.7 vs 37.4\%). ${ }^{27}$ Significantly fewer patients required vitrectomy at 6 months after treatment with ocriplasmin than with placebo (17.7 vs $26.6 \%$; $P=0.02$ ). In patients not undergoing subsequent vitrectomy, an improvement in best-corrected VA (BCVA) of three or more Snellen lines was observed in $9.7 \%$ of eyes following ocriplasmin treatment and in $3.5 \%$ of eyes following placebo treatment $(P=0.008) .{ }^{27}$

The most common ocular adverse events reported in these studies were vitreous floaters (ocriplasmin, 16.8 vs placebo, $7.5 \%$; $P=0.002)$, conjunctival haemorrhage (14.6 vs $12.8 \% ; P=0.53)$, injection-related eye pain (13.5 vs $5.9 \% ; P=0.005)$, and photopsia (11.8 vs $2.7 \% ; P<0.001) .^{27}$ These were mostly mild and transient. Serious adverse events were $\mathrm{MH}$ development (5.2 vs 8.6\%; $P=0.15)$, retinal detachment $(0.4$ vs $1.6 \% ; P=0.16)$, and reduced VA ( 0.6 vs $0.5 \% ; P=0.94)$, with no significant differences between ocriplasmin and placebo. ${ }^{27}$

Some data are now available for the use of ocriplasmin in clinical practice (Table 1). These demonstrate successful VMA resolution in $66-80 \%$ of eyes with $\mathrm{VMT}+\mathrm{MH}$ and in 50-62\% of eyes with $\leq \mathrm{VMA}$ $1500 \mu \mathrm{m} .^{28-31}$

There have been reports of changes to the photoreceptor outer segment ellipsoid zone on optical coherence tomography (OCT) following injection with ocriplasmin in clinical practice. ${ }^{31-34}$ The largest retrospective study reported that 7 out of 17 patients injected with ocriplasmin experienced ellipsoid zone loss on spectral domain OCT (SD-OCT). ${ }^{31}$ The loss of the OS ellipsoid zone was transient in all patients, with an average time to loss of 5 days and an average time to return of 29.3 days. ${ }^{31}$ In addition, there have been individual case reports of severe vision loss post injection with ocriplasmin, as well as vision dimming and ERG changes. ${ }^{26,31,33,34}$ These are symptoms and side effects that have been noted in the MIVI TRUST studies and documented within the European Summary of Product Characteristics for ocriplasmin; therefore, it is important that patients are made aware of both the risks and their associated prognoses.

\section{NICE guidance for ocriplasmin}

\section{NICE guidance}

The National Institute of Health and Care Excellence (NICE) recommendations regarding the use of ocriplasmin state that it is an option for the following patients with VMT: no ERM is present, and a stage $2 \mathrm{MH}$ is present (with a diameter of $\leq 400 \mu \mathrm{m}$ ), and/or they have severe symptoms. ${ }^{35}$ Symptom severity is not quantified in the NICE guidance but it is suggested that patients with metamorphopsia and low VA causing them distress would be eligible for ocriplasmin treatment. ${ }^{35}$

The NICE guidance states that ocriplasmin is costeffective for patients with VMT and no ERM, and those with VMT with stage $2 \mathrm{MH} .{ }^{35}$ However, the primary challenge for Clinical Commissioning Groups (CCGs) is to implement the NICE guidance within existing cost and capacity constraints. In order to fund new therapies CCGs must make savings in other areas (eg, decommissioning another treatment). Lack of capacity is also a key issue, and this could impact upon intervals between follow-up visits.

\section{Patient population}

In order to allocate budgets for the usage of ocriplasmin in VMT/MH patients within the National Health Service (NHS) in England and Wales, CCGs will require a realistic estimate of the number of patients who are likely to receive this treatment. However, there are inherent difficulties in categorising and quantifying the potential VMT population prior to the availability of this treatment. Although audit data are available for VMT patients referred for vitrectomy, these do not capture the full range of patients who might receive ocriplasmin. For example, some patients with severe symptomatic VMT 
Table 1 Ocriplasmin in clinical practice: patients achieving vitreomacular adhesion resolution

\begin{tabular}{lccccccc}
\hline Institute & Patients & FTMH present & FTMH absent & ERM present & ERM absent & VMA $\leq 1500 \mu m$ & VMA $>1500 \mu m$ \\
\hline Bascom Palmer Eye Institute $^{28}$ & 19 & $4 / 6(66.7 \%)$ & $4 / 13(30.8 \%)$ & $2 / 8(50.0 \%)$ & $5 / 11(45.0 \%)$ & $8 / 16(50.0 \%)$ & $0 / 3(0)$ \\
NJ Retina and Vitreous Centre $^{29}$ & 52 & $11 / 15(73.3 \%)$ & $7 / 37(19.0 \%)$ & $1 / 11(9.0 \%)$ & $17 / 41(41 / 5 \%)$ & NR $(38.0 \%)^{\mathrm{a}}$ & NR $(0)^{\mathrm{b}}$ \\
California Retina Research Foundation $^{30}$ & 25 & $8 / 11(72.7 \%)$ & $6 / 14(42.9 \%)$ & $13 / 23(56.5 \%)$ & $1 / 2(50.0 \%)$ & NA & NA \\
Cole Eye Institute $^{31}$ & 17 & $2 / 2^{\mathrm{c}}(100.0 \%)$ & $6 / 14(42.9 \%)$ & $1 / 3(33.3 \%)$ & $7 / 14(50.0 \%)$ & $8 / 13(61.5 \%)$ & $0 / 4(0)$ \\
\hline
\end{tabular}

Abbreviations: ERM, epiretinal membrane; FTMH, full-thickness macular hole; VMA, vitreoretinal adhesion; NA, not applicable; NR, not reported. aVMA $\leq 750 \mu \mathrm{m}$.

${ }^{b} \mathrm{VMA}>751 \mu \mathrm{m}$

'Includes Stage 2 macular holes only, as per licenced indication for ocriplasmin. ${ }^{26}$

could receive ocriplasmin at an earlier stage in the disease process - that is, before they would normally be offered surgery. The population receiving ocriplasmin could also include a subgroup of patients who have refused surgery. However, some patients choosing not to undergo surgery may also refuse the offer of treatment with ocriplasmin, preferring instead to live with their symptoms.

Perhaps most importantly, there is a risk of underestimating the number of patients eligible to receive ocriplasmin and this has obvious financial implications. Such an issue has been observed in patients with neovascular (wet) age-related macular degeneration (wAMD). Estimates for the prevalence of wAMD have increased substantially. A meta-analysis published in 2012 estimated a prevalence of 263000 cases of WAMD in the United Kingdom, compared with 245000 in $2003 .{ }^{36,37}$ The number of patients with wAMD who would be eligible for treatment has risen from an estimated 7000 in 2003 to 26000 per year, because of availability of novel therapies. ${ }^{37}$ Currently, it has been estimated that $10-20$ patients per 100000 could represent the annual potential population eligible for treatment with ocriplasmin (authors' own estimate).

\section{Treatment administration and handling}

It has been proposed that ocriplasmin should routinely be administered in a dedicated clean room. However, both theatres and clean rooms may have capacity issues, as the number of intravitreal injections administered in England rose from 10.7/100 000 in 2006-2007 to $59.5 / 100000$ in $2008-2009 .{ }^{38}$ Use of a theatre may be feasible if an individual institution expects to treat a low number of patients.

There are risk management issues to consider when using a clean room. One approach to address this is having dedicated lists for ocriplasmin administration. Guidelines published by the Royal College of Ophthalmologists (RCOphth) in 2009 provide a framework for administering intravitreal injections, including requirements for clean rooms. These also state that intravitreal injections should always be administered by qualified and trained ophthalmic surgeons, who have experience of this mode of treatment administration and management of intravitreal injection-related complications (or by a trainee under supervision of such an ophthalmologist). ${ }^{39}$

As ocriplasmin is a 'frozen chain' product, it is important to consider the correct storage and transportation requirements. It must be stored at $-20^{\circ} \mathrm{C}$ and prepared immediately before use. ${ }^{26}$ It takes approximately $2 \mathrm{~min}$ to defrost, and after dilution it should be injected as soon as possible in order to maximise efficacy. Prior to handling ocriplasmin (removing it from the freezer), it should be ensured that the patient is present and their OCT images have been checked.

\section{The patient pathway}

\section{Current options}

Currently, the key factors determining the pathway for patients with suspected VMT + MH include local sharedcare agreements between primary and secondary care providers, and the availability of both medical retina (MR) specialists and vitreoretinal (VR) surgeons. Such specialists may therefore receive patient referrals via a number of routes, including from optometrists, other hospital departments/specialities, and from Accident and Emergency departments. VMT patients without MH are usually referred on the basis of self-reported symptoms - for example, metamorphopsia.

Subsequently, MR specialists generally refer patients with suspected VMT + MH to VR surgeons. However, patients with a short duration of VMT-associated symptoms may not be included in surgical guidelines, and these may be monitored in the MR clinic every 3-6 months. If an MR specialist anticipates that a patient's condition will progress more quickly, they are asked to return in 1-2 months after the initial visit. In general, patients with VMT and no MH are managed by observation only, whereas patients with VMT $+\mathrm{MH}$ are more likely to undergo vitrectomy. 
A VR service has been set up at the University Hospital of North Staffordshire, which includes the development of a template that is issued to optometrists and General Practitioners in order to help them prioritise acute referrals. In addition, training was provided for optometrists who had a specific interest in retinal pathologies (OPSIs). An MR or VR specialist reviews any OCT images sent from optometrists to decide whether a patient needs to be referred for treatment or observed for a certain period of time. However, it has been noted that OCT findings do not always reflect the patient's subjective experience, and cases of asymptomatic VMT identified via OCT have been reported. Asymptomatic patients are given information to help them self-monitor their condition and are asked to return if symptoms develop. Such symptoms may include a decrease in VA, metamorphopsia, and so on.

Virtual clinics have been piloted in Fife and Sunderland. These utilise remote digital imaging screening, but the need to invest in IT systems for largescale use presents a significant barrier to translating the potential benefits into everyday practice. Most optometrists are unable to send compressed OCT images for screening as they do not have NHS secure networks, which could give rise to further problems such as patient confidentiality and the potential to miss important abnormalities. However, if local optometrists have negotiated access to the nhs.net email system using an N3 node sited at a local GP practice, the confidentiality issue is reduced.

\section{Stage 1: referral}

To address the existing challenges of appropriate and timely referrals of VMT/MH patients and to incorporate ocriplasmin as an option for certain subgroups, a revised pathway for diagnosis, assessment, and treatment is needed. However, existing local pathways should be taken into account when considering the redesign of a VMT service. It is important that MR specialists and VR surgeons work closely together with primary care stakeholders when revising the service they offer. Ophthalmic nurses should also be an integral member of the multidisciplinary team, as they may act as a bridge between primary and secondary care, for example, triaging patients in A\&E and referrals from GPs, and be able to provide counselling to patients and their families. ${ }^{40}$

Under shared-care agreements in some areas, OPSIs play an important role in screening referrals to secondary care. In Hereford, for example, the Glaucoma Referral Refinement Pathway has been successful in reducing the number of unnecessary referrals to secondary care, which is associated with cost savings. Therefore, there is potential for OPSIs to perform OCT imaging as part of the VMT pathway. Indeed, if OCT evidence is mandatory for referrals, this may allow some patients to continue being managed in primary care-for example, if the patient has symptoms but the OCT demonstrates normal VR anatomy. However, OPSIs must be trained to be confident in distinguishing between VMA and VMT. The College of Optometrists has released a Higher Qualification in MR management to complement those already available for low vision, glaucoma, independent prescribing, and so on.

\section{Stage 2: VMT triage clinic}

A VMT triage clinic (or VMT referral centre) could be set up, with the aim of managing patients requiring treatment (including those eligible for ocriplasmin) more efficiently (Figure 2). However, to take into account the existing local variations, local ophthalmology committees must be involved from the outset in the development of a new pathway. A key issue will be the nomination of a VMT triage clinic.

Patient assessment should include OCT and an accurate measurement of BCVA using the Early Treatment of Diabetic Retinopathy Study (ETDRS) chart. Time-domain OCT (TD-OCT) allows a maximum axial resolution of approximately $8-10 \mu \mathrm{m}$, whereas SD-OCT demonstrates an axial resolution of $5-8 \mu \mathrm{m} .^{4,41,42} \mathrm{In}$ clinical practice, this means that, although TD-OCT permits visualisation of the VR interface, imaging of the posterior hyaloid face is limited. ${ }^{4,43}$ In contrast, SD-OCT allows accurate visualisation of both the posterior hyaloid face and the choroid. ${ }^{44}$ In addition, when threedimensional SD-OCT is employed, identification of ERM and quantification of VMT diameter, retinal thickness, and $\mathrm{MH}$ dimensions is possible, which can have an impact on treatment decision-making and procedural approaches. ${ }^{20,43,44}$ Other investigations, such as fundus fluorescein angiography and microperimetry, are generally not required. It should not be assumed that all optometrists have access to OCT, although many do and the numbers are increasing. Currently, there is no central guidance for optometrists on the referral criteria with regard to OCT. Agreement of a fixed cost for OCT (including clinical time to review and report results of images) would facilitate decision-making regarding the location of where it is performed - that is, primary or secondary care.

\section{Stage 3: clinics and treatment decisions}

It has been proposed that patients with VMT $+\mathrm{MH}$ should be managed as a routine 6-week referral (unlike wAMD, which requires a fast-track clinic). This reflects 


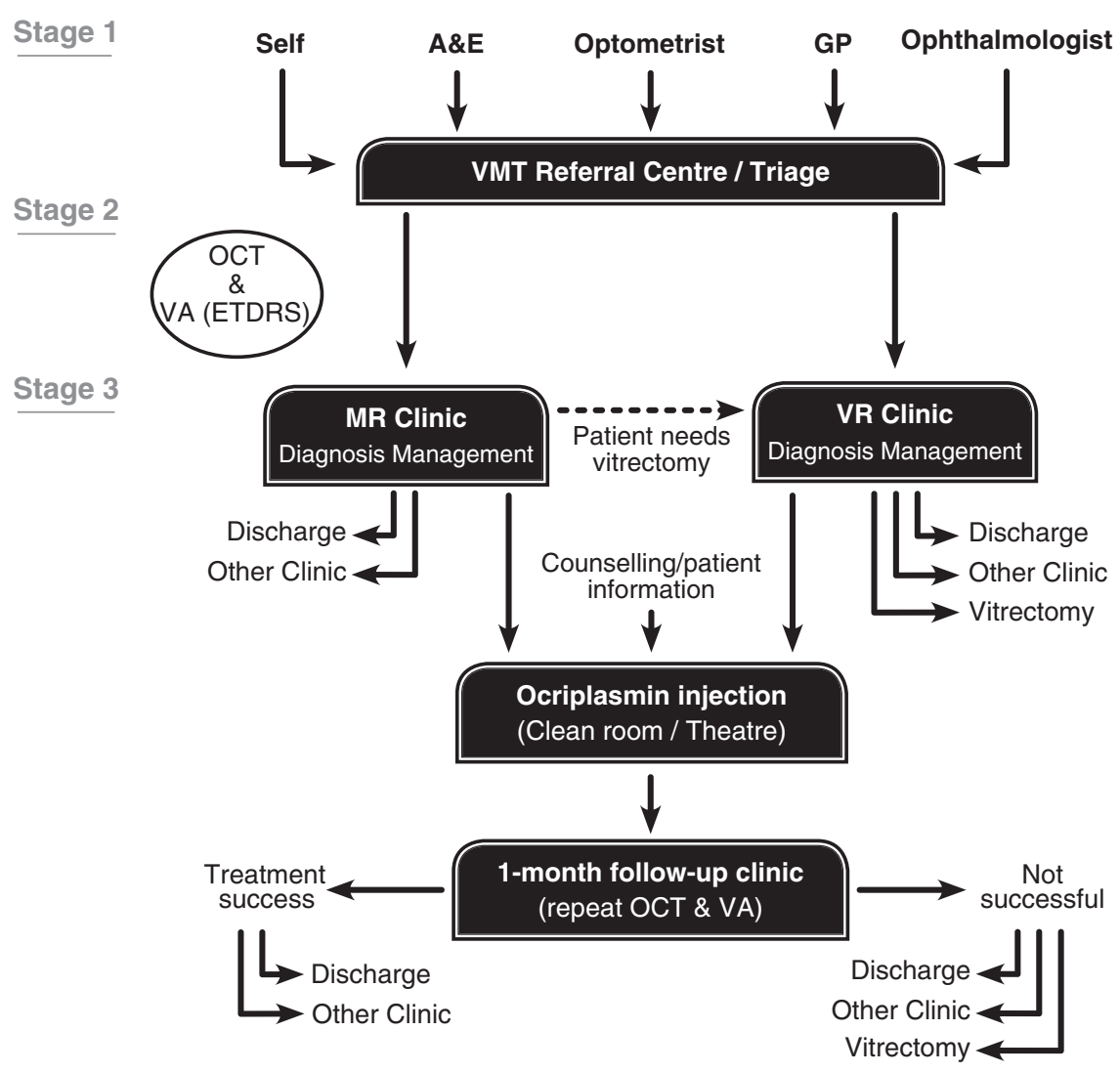

Figure 2 A redesigned pathway for vitreomacular traction and macular hole management, with the incorporation of ocriplasmin. A\&E, Accident and Emergency; EDTRS, Early Treatment of Diabetic Retinopathy Study; GP, general practitioner; MR, medical retina; OCT, optical coherence tomography; VA, visual acuity; VMT, vitreomacular traction; VR, vitreoretinal.

the patient population of the two Phase III clinical trials for ocriplasmin, which showed that the mean interval between diagnosis and entering the trial was 62 days for $\mathrm{VMT}+\mathrm{MH}$ and 260-270 days for VMT (with and without ERM). ${ }^{45}$ Scheduling up to two hospital visits is a feasible and realistic approach for VMT/MH patients. Ideally, the MR specialist or VR surgeon would decide at the first visit whether or not treatment with ocriplasmin is appropriate and feasible, and the injection would be administered at the second visit. However, there is local variation within ophthalmology units as to whether or not MR specialists and VR surgeons are both present. The proposed redesigned referral pathway includes automatic referral to a VR specialist if a patient is considered to require surgery. The proposed redesigned pathway also includes a clinic appointment at 1 month after ocriplasmin injection, to assess treatment outcome based on follow-up OCT and VA measurements. This should ideally be performed at the treatment centre.

Patient eligibility for ocriplasmin should be reviewed according to the product licence, NICE guidance, and available clinical evidence. For example, the absence of ERM at baseline may enhance the likelihood of treatment success, according to the pre-specified subgroup analysis of data from the two Phase III clinical trials. ${ }^{27}$ Other parameters, such as VMA width, may also be predictive of successful ocriplasmin treatment, as indicated by a post-hoc analysis of clinical trial data and recently reported clinical practice findings (Table 1). ${ }^{28-31,46} \mathrm{In}$ accordance with NICE guidance, patients with isolated severe symptomatic VMT (ie, without MH) can now receive ocriplasmin before disease progression, for example, the formation of a $\mathrm{MH}$, whereas previously these patients were predominantly managed with observation only. ${ }^{35}$

The complete patient journey - from initial referral to treatment (including surgery after ocriplasmin if required) - should take no longer than 6 months, on the basis that outcomes for VMT or VMT + MH may be worse if surgery is delayed by more than this length of time. ${ }^{10,11,23}$

It is therefore important that the administration of ocriplasmin should not delay surgery if it is subsequently required. Indeed, NICE recommends that, in patients with stage II MH, ocriplasmin be used during the wait for surgery without delaying the procedure. ${ }^{32}$ The 
RCOphth College Statement regarding ocriplasmin also notes the need for local management pathways to ensure ocriplasmin use does not delay surgery for patients not achieving the expected outcome of $\mathrm{MH}$ closure. ${ }^{47}$ CCGs should be aware of the proposed revised pathway, especially with respect to timings and the possibility of vitrectomy following ocriplasmin.

Prior to the introduction of ocriplasmin into a centre, pharmacists must be involved in the decision-making regarding the practical aspects of its use-for example, purchase, storage (the need for freezer storage, proximity to the administration setting), and reconstitution in accordance with local standard operating procedures.

\section{Audit of the redesigned service}

Patient follow-up data should be collected by treatment centres for 1 year to evaluate the new VMT service, and to allow continued improvements. Quality-of-life outcomes could be collected to build support for ocriplasmin treatment in eligible patients. For example, if a treatment means that a patient can continue their activities of daily living, then capturing these outcomes will support an argument for spending money now (on a treatment) in order to make future savings (eg, from social care budgets). However, simple tools would be needed to capture this information in everyday clinical practice.

\section{Patient considerations}

The goals of therapy are currently different for VMT patients with and without MH. In VMT patients with metamorphopsia, the current goals of therapy are symptom improvement and long-term preservation of vision. In VMT patients with small $\mathrm{MH}$, the current expectation is that VA will improve in the majority of patients after vitrectomy.

The risks and benefits of vitrectomy should be discussed with patients, as appropriate. ${ }^{48}$ In line with RCOphth guidelines regarding intravitreal injections, ophthalmology clinicians should explain the importance of treatment, why ocriplasmin is appropriate for them, the expected outcomes and possible side effects to patients, at the treatment decision stage. ${ }^{39,48}$ This includes what action to take in the event of certain postinjection complications. ${ }^{48}$ More detailed information is provided in the form of leaflets for patients to take away. ${ }^{49}$

Success with ocriplasmin is expected in up to $50 \%$ of VMT patients with $\mathrm{MH}$ and up to $37.4 \%$ of VMT patients without $\mathrm{MH}$ (and without ERM). ${ }^{27,45}$ A recently published series of 19 cases of patients receiving
Table 2 Checklist for redesign of a VMT service

- Review existing local pathways with local ophthalmology committees

- Nominate a VMT referral centre/triage clinic

- Decide who will perform OCT

- Provide guidance/training for referrers (GPs, A\&E, optometrists, ophthalmology nurses)

- Decide how diagnostic information, ie, OCT will be transferred from referrer to specialist

- Designate the treatment setting and logistics for ocriplasmin storage and administration

- Schedule assessment visit, ocriplasmin administration visit, follow-up appointments

- Add patients to vitrectomy list even if they are receiving ocriplasmin

- Provide information/counselling for patients

- Design an audit process and define information to be captured

ocriplasmin reported VMA resolution in $42 \%$ of patients and $\mathrm{MH}$ closure in $50 \%$ of patients. ${ }^{28}$

The risks and details of the potential side effects of ocriplasmin should be reiterated on the day of injection. For example, patients may experience symptoms such as vitreous floaters and photopsia, which can indicate that the process of PVD has begun. In the Phase III studies, $7.7 \%$ of ocriplasmin patients and $1.6 \%$ of placebo patients had acute transient $\geq 2$-line ( $\geq 10$ ETDRS letters) loss in BCVA during the first week after injection with no alternative explanation for the change. ${ }^{26}$ VA decreases were generally reversible within 2 weeks without intervention.

\section{Summary}

There are local variations in how VMT patients are currently managed. Managing referrals from optometrists through a VMT triage clinic would therefore improve efficiency of the service, with the aim of minimising inappropriate referrals to MR clinics and/or VR surgeons (it is crucial that the distinction between VMA and VMT is made when assessing OCT images). A fixed cost for OCT imaging as part of the VMT/MH treatment pathway would allow local flexibility on where to carry out the assessment.

The goals of therapy are currently different for VMT patients with and without $\mathrm{MH}$. It has been established that outcomes of vitrectomy are less favourable when performed more than 6 months after diagnosis. However, the introduction of ocriplasmin may allow early treatment of symptomatic VMT and $\mathrm{MH}$ before these conditions progress. NICE guidance recommends that ocriplasmin treatment be administered while patients are awaiting vitrectomy, so that these procedures are not delayed in the case of treatment failure. 
The decision to use ocriplasmin should be based on NICE guidance in order to be supported by CCGs. It is proposed that ocriplasmin ideally be administered in a clean room, although capacity may be an issue. Patients will need appropriate counselling about the expected outcomes and possible side effects of ocriplasmin treatment. One-year follow-up data should be collected by treatment centres in order to evaluate the new VMT service.

A suggested checklist for VMT service redesign is presented in Table 2 .

\section{Conflict of interest}

Winfried Amoaku has received honoraria for participation in advisory board meetings sponsored by Alcon, Allergan, Novartis, and Thrombogenics, for presentations sponsored by Alcon, Alimera Science, Allergan, Novartis, and Thrombogenics, and holds research grants from Allergan and Novartis. Peter Cackett has received honoraria for participation in advisory board meetings sponsored by Alcon, Bayer, and Novartis, and from Novartis for presentations. Ajai Tyagi has received honoraria for participating in advisory boards and speaking engagements sponsored by Alcon, and his employer has received a grant from Alcon. Usman Mahmood declares no conflict of interest. Jennifer Nosek has received honoraria for participation in advisory board meetings sponsored by Alcon, Alimera Science, Bayer, and Novartis. Graham Mennie has received honoraria for participation in advisory board meetings sponsored by Alcon, Bayer, and Novartis. Nicholas Rumney has received an honorarium for participation in an advisory board meeting sponsored by Alcon.

\section{Acknowledgements}

This supplement has been funded by Alcon Eyecare UK, with medical writing provided on their behalf by 90TEN Healthcare. Alcon Eyecare UK has had the opportunity to review the article for technical accuracy but has had no editorial input.

\section{References}

1 Jackson TL, Nicod E, Simpson A, Angelis A, Grimaccia F, Kanavos P. Symptomatic vitreomacular adhesion. Retina 2013; 33: 1503-1511.

2 La Cour M, Friis J. Macular holes: classification, epidemiology, natural history and treatment. Acta Ophthalmol Scand 2002; 80: 579-587.

3 Johnson M. Perifoveal vitreous detachment and its macular complications. Trans Am Ophthalmol Soc 2005; 103: 537-567.

4 Barak Y, Ihnen MA, Schaal S. Spectral domain optical coherence tomography in the diagnosis and management of vitreoretinal interface pathologies. J Ophthalmol 2012; 2012: 1-7.
5 Ezra E. Idiopathic full thickness macular hole: natural history and pathogenesis. Br J Ophthalmol 2001; 85: 102-108.

6 Sebag J. Anatomy and pathology of the vitreo-retinal interface. Eye 1992; 6: 541-552.

7 Knudtson MD, Klein BEK, Klein R, Cruickshanks KJ, Lee KE. Age-related eye disease, quality of life, and functional activity. Arch Ophthalmol 2005; 123: 807-814.

8 Okamoto F, Okamoto Y, Fukuda S, Hiroka T, Oshika T. Vision-related quality of life and visual function after vitrectomy for various vitreoretinal disorders. Invest Ophthalmol Vis Sci 2010; 51: 744-751.

9 McCannel CA, Ensminger JL, Diehl NN, Hodge DN. Population based incidence of macular holes. Ophthalmology 2009; 116: 1366-1369.

10 Ip MS, Baker BJ, Duker JS, Reichel E, Baumal CR, Gangnon R et al. Anatomical outcomes of surgery for idiopathic macular hole as determined by optical coherence tomography. Arch Ophthalmol 2002; 120: 29-35.

11 Jaycock PD, Bunce C, Xing W, Thomas D, Poon W, Gazzard G et al. Outcomes of macular hole surgery: implications for surgical management and clinical governance. Eye 2005; 19: 879-884.

12 Nicod E, Jackson TL, Grimaccia F, Angelis A, Kanavos P. Cost analysis of pars plana vitrectomy for the treatment of symptomatic vitreomacular adhesion: a bottom-up costing perspective. Presented at the ISPOR $18^{\text {th }}$ Annual International Meeting, New Orleans, 20-22 May, 2013. Value Health 2013; 16: A176. Abstract PSS6.

13 Steel DHW, Lotery AJ. Idiopathic vitreomacular traction and macular hole: a comprehensive review of pathophysiology, diagnosis and treatment. Eye 2013; 27: S1-S27.

14 Hickichi T, Yoshida A, Trempe CL. Course of vitreomacular traction syndrome. Am J Ophthalmol 1995; 119: 55-61.

15 Sonmez K, Capone Jr A, Trese MT, Williams GA. Vitreomacular traction syndrome: impact of anatomical configuration on anatomical and visual outcomes. Retina 2008; 28: 1207-1214.

16 Witkin AJ, Patron ME, Castro LC, Reichel E, Rogers AH, Baumal CR et al. Anatomic and visual outcomes of vitrectomy for vitreomacular traction syndrome. Ophthalmic Surg Lasers Imaging 2010; 41: 425-431.

17 Chew EY, Sperduto RD, Hiller R, Nowroozi L, Seigel D, Yanuzzi LA et al. Clinical course of macular holes. The Eye Disease Case-control Study. Arch Ophthalmol 1999; 117: 242-246.

18 Hickichi T, Yoshida A, Akiba J, Trempe CL. Natural outcomes of stage 1, 2, 3 and 4 idiopathic macular holes. Br J Ophthalmol 2005; 79: 517-520.

19 Kang SW, Ahn K, Ham D-I. Types of macular hole closure and their clinical implications. Br J Ophthalmol 2003; 87: 1015-1019.

20 Wakely L, Rahman R, Stephenson J. A comparison of several methods of macular hole measurement using optical coherence tomography, and their value in predicting anatomical and visual outcomes. Br J Ophthalmol 2012; 96: 1003-1007.

21 Jackson TL, Donachie PHJ, Sparrow JM, Johnston RL. United Kingdom National Ophthalmology Database Study of Vitreoretinal Surgery: report 2, macular hole. Ophthalmology 2013; 120: 629-634.

22 Kang HK, Chang AA, Beaumont PE. The macular hole: report of an Australian surgical series and meta-analysis of the literature. Clin Exp Ophthalmol 2000; 28: 298-308.

23 Theodossiadis GP, Grigoropoulos VG, Theodoropolou S, Datseris I, Theodossiadis PG. Spontaneous resolution of 
vitreomacular traction demonstrated by spectral-domain optical coherence tomography. Am J Ophthalmol 2014; 157: 842-851.

24 Passemard M, Yakoubi Y, Muselier A, Hubert I, Guillaubey A, Bron AM et al. Long-term outcome of idiopathic macular hole surgery. Am J Ophthalmol 2010; 149: 120-126.

25 Jackson TL, Donachie PH, Sparrow JM, Johnston RL. United Kingdom National Ophthalmology Database Study of Vitreoretinal Surgery: report 1; case mix, complications, and cataract. Eye 2013; 27: 644-651.

26 Jetrea $0.5 \mathrm{mg} / 0.2 \mathrm{ml}$ concentrate for solution for injection. Summary of Product Characteristics, March 2013.

27 Stalmans P, Benz MS, Gandorfer A, Kampik A, Girach A, Pakola $S$ et al. Enzymatic vitreolysis with ocriplasmin for vitreomacular traction and macular holes. $N$ Engl J Med 2012; 367: 606-615.

28 Kim BT, Schwarz SG, Smiddy WE, Doshi RR, Kovach JL, Berrocal AM et al. Initial outcomes following intravitreal ocriplasmin for treatment of symptomatic vitreomacular adhesion. Ophthalmic Surg Lasers Imaging Retina 2013; 44: 334-343.

29 Roth D.. Ocriplasmin for the treatment of vitreomacular traction: clinical indications and predictors of success. Presentation at the 31st Annual Meeting of the American Society of Retina Specialists, Toronto, August 24-28, 2013;

30 Nasir MA, Pieramici DJ, Castellarin AA, See R, Couvillion S, Steinle NC et al. Intravitreal ocriplasmin for vitreomacular adhesion syndrome: initial experience of multi-physician retina practice. Poster presented at the 31st Annual Meeting of the American Society of Retina Specialists, Toronto, August 24-28, 2013.

31 Singh RP, Li A, Bedi R, Srivastava S, Sears JE, Ehlers JP et al. Anatomical and visual outcomes following ocriplasmin treatment for symptomatic vitreomacular traction syndrome. $\mathrm{Br}$ J Ophthalmol 2014; 98: 356-360.

32 Freund KB, Shah SA, Shah VP. Correlation of transient vision loss with outer retinal disruption following intravitreal ocriplasmin. Eye 2013; 27: 773-774.

33 Tibbetts MD, Reichel E, Witkin AJ. Vision loss after intravitreal ocriplasmin: correlation of spectral-domain coherence tomography and electroretinography. JAMA Ophthalmol 2014; 132: 487-490.

34 Fahim AT, Khan NW, Johnson MW. Acute panretinal structural and functional abnormalities after intravitreous ocriplasmin injection. JAMA Ophthalmol 2014; 132: 484-486.

35 National Institute for Health and Care Excellence. Ocriplasmin for treating vitreomacular traction. NICE technology appraisal guidance 297. October 2013;

36 Owen CG, Fletcher AE, Donoghue M, Rudnicka AR. How big is the burden of visual loss caused by age-related macular degeneration in the United Kingdom? Br J Ophthalmol 2003; 87: 312-317.

\section{Appendix}

JETREA ${ }^{\circledR} 0.5 \mathrm{mg} / 0.2 \mathrm{ml}$ concentrate for solution for injection (ocriplasmin) Prescribing Information. (Refer to full Summary of Product Characteristics (SmPC) before prescribing).

Presentation: Type I glass vial containing $0.2 \mathrm{ml}$ concentrate for solution for injection. After dilution
37 Owen CG, Jarrar Z, Wormald R, Cook DG, Fletcher AE, Rudnicka AR. The estimated prevalence and incidence of late stage age-related macular degeneration in the UK. Br J Ophthalmol 2012; 96: 752-756.

38 Keenan TD, Wotton CJ, Goldacre MJ. Trend over time and geographical variation rates of intravitreal injections in England. Br J Ophthalmol 2012; 96: 413-418.

39 Guidelines for intravitreal injections procedure 2009. The Royal College of Ophthalmologists, London 2009.

40 The nature, scope and value of ophthalmic nursing 3rd ed. Royal College of Nursing: London 2009.

41 Drexler W, Fujimoto JG. State-of-the art retinal coherence tomography. Prog Retin Eye Res 2008; 27: 45-88.

42 Sull AC, Vuong LN, Price LL, Srinivasan VJ, Gorczynska I, Fujimoto JG et al. Comparison of spectral/Fourier domain optical coherence tomography instruments for assessment of normal macular thickness. Retina 2010; 30: 235-245.

43 Koizumi H, Spaide RF, Fisher YL, Freund KB, Klancnik Jr JM, Yannuzzi LA. Three-dimensional evaluation of vitreomacular traction and epiretinal membrane using spectral-domain optical coherence tomography. Am J Ophthalmol 2008; 145: 509-517.

44 Adhi M, Duker JS. Optical coherence tomography - current and future applications. Curr Opin Ophthalmol 2013; 24: 213-221.

45 Narendran N, Yang Y, Brittain C, Lescrauwaet B. Baseline characteristics across clinically relevant subgroups of vitreomacular traction patients enrolled in ocriplasmin phase 3 randomised controlled trials. Poster presented at the 13th International AMD and Retina Congress, Dublin, 25-26 October, 2013.

46 Kupperman B. Baseline features predictive of pharmacologic vitreomacular adhesion (VMA) resolution in the ocriplasmin MIVI-TRUST program. Congress of the European Society of Ophthalmology, Copenhagen, Denmark, June 8-11, 2013. Abstract FP-RET-092.

47 College statement. Ocriplasmin intravitreal injection for the treatment of vitreomacular traction The Royal College of Ophthalmologists: London 2013.

48 Ophthalmic services guidance. Ophthalmic Daycare and Inpatient Facilities. The Royal College of Ophthalmologists: London 2012.

49 Ophthalmic services guidance. Ophthalmic Outpatient Department. The Royal College of Ophthalmologists: London 2012.

This work is licensed under the Creative Commons Attribution-NonCommercial-No Derivative Works 3.0 Unported License. To view a copy of this license, visit http://creativecommons.org/licenses/ by-nc-nd/3.0/

with $0.2 \mathrm{ml}$ sodium chloride $0.9 \%$ solution for injection, $0.1 \mathrm{ml}$ of the diluted solution contains $0.125 \mathrm{mg}$ ocriplasmin.

Indication(s): Treatment of vitreomacular traction (VMT) in adults, including when associated with macular hole of diameter less than or equal to 400 microns.

Posology and method of administration: Adults, including the elderly: Intravitreal injection of $0.125 \mathrm{mg}$ 
( $0.1 \mathrm{ml}$ of the diluted solution) to the affected eye once only as a single dose.

Children and adolescents: Not recommended.

Hepatic and renal impairment: No dosage adjustment necessary.

Contra-indications: Hypersensitivity to ocriplasmin or any of the excipients. Active or suspected ocular or periocular infections.

Warnings and precautions: Proper aseptic injection techniques must always be used and patients should be monitored for any side effects such as intraocular inflammation/infection and elevation in IOP. Patients should be instructed to report symptoms of intraocular inflammation/infection or any other visual/ocular symptoms without delay. Concurrent administration to both eyes or repeated administration in the same eye is not recommended. Clinical data are not available on concomitant use with VEGF-inhibitors. Treatment is not recommended in patients with large diameter macular holes, high myopia, aphakia, history of rhegmatogenous retinal detachment, lens zonule instability, recent ocular surgery or intraocular injection (including laser therapy), proliferative diabetic retinopathy, ischaemic retinopathies, retinal vein occlusions, exudative age-related macular degeneration (AMD), and vitreous haemorrhage. There is potential for lens subluxation or phacodonesis. Exercise caution if treating patients with nonproliferative diabetic retinopathy, history of uveitis, or significant eye trauma. Ocriplasmin efficacy is reduced in patients with an epiretinal membrane or a diameter of VMA > 1500 microns. Due to potential increase in tractional forces, there is a risk of occurrence of new or enlarged macular holes. There is a risk of significant but transient loss of visual acuity during the first week after the injection.

Interactions: Formal studies have not been performed. Systemic interactions are not anticipated. Administration in close temporal association, in the same eye as other medicinal products, may affect the activity of both products and is not recommended.

Pregnancy and lactation: Do not use unless clearly necessary. Fertility: No fertility data are available.
Effects on ability to drive and use machines: If visual disturbances occur, wait until vision clears before driving or operating machinery.

Undesirable effects: Very common: vitreous floaters, eye pain, conjunctival haemorrhage. Common: visual acuity reduced, visual impairment, vision blurred, intraocular pressure increased, metamorphopsia, conjunctival oedema, eyelid oedema, photopsia, conjunctival hyperaemia, ocular hyperaemia, retinogram abnormal, eye irritation, dry eye, foreign body sensation in eyes, eye pruritus, ocular discomfort, photophobia, chromatopsia. Serious: retinal haemorrhage, vitreous haemorrhage, retinal tear, retinal detachment, macular hole, macular degeneration, retinal degeneration, macular oedema, retinal oedema, retinal pigment epitheliopathy, vitreous adhesions, vitritis, anterior chamber cell, anterior chamber flare, iritis, vitreous detachment, transient blindness, lens subluxation, scotoma, visual field defect, hyphaema, pupils unequal, corneal abrasion, anterior chamber inflammation. In addition: diplopia, miosis, eye inflammation, conjunctival irritation. Prescribers should consult the $\mathrm{SmPC}$ in relation to the side effects.

Overdose: Clinical data are limited. If an overdose occurs, close monitoring is recommended.

Incompatibilities: Must not be mixed with other medicinal products except for the specified diluent.

Special precautions for storage: Store frozen at $-20^{\circ} \pm 5^{\circ} \mathrm{C}$.

Legal category: POM.

Package quantities and basic NHS costs: $1 \times 0.2 \mathrm{ml}$ vial $£ 2,500$ Ireland price: $€ 3099$.

MA number(s): EU/1/13/819/001.

Further information available from the MA holder: ThromboGenics NV, Gaston Geenslaan 1, B-3001 Leuven, Belgium.

Distributor: Alcon Laboratories (UK) Limited, Frimley Business Park, Frimley, Camberley, Surrey GU16 7SR, UK.

Date of preparation: June 2013 (V2)

Adverse events should be reported. Reporting forms and information can be found at www.mhra.gov.uk/ yellowcard. Adverse events should also be reported to Alcon Medical Information. Tel: 0871376 1402. Email: gb. adr@alcon.com 